\title{
Ecocrítica alemã: Um panorama
}

[German Ecocriticism: An overview]

http://dx.doi.org/10.1590/1982-8837119

\section{Axel Goodbody ${ }^{1}$}

\begin{abstract}
The article presents the state of art of ecocritical research on German literature. Although it has started in a later period in comparison to the USA, there is already a number of publications that indicate that this is a developing research field.
\end{abstract}

Key-words: ecocriticism; environment; German literature

Resumo: O artigo apresenta o estado da arte da pesquisa ecocrítica sobre a literatura alemã. Apesar de esta ter se iniciado tardiamente em comparação com os EUA, já há um número de publicações que apontam para uma área em desenvolvimento.

Palavras-chave: ecocrítica; meio-ambiente; literatura alemã

\section{Introdução da tradutora}

Apresentamos uma versão estendida do panorama sobre os estudos ecocríticos em literatura alemã de Axel Goodbody publicado em The Oxford Handbook of Ecocriticism (ed. Greg Garrard) em 2014. O texto apresenta o estado da arte da pesquisa ecocrítica em literatura alemã. Como é apontado pelo autor, apesar de a Alemanha se destacar no debate ecológico tanto pela filosofia quanto pela consciência de seus habitantes, os estudos na área de literatura foram esquecidos por um longo período ou relegados ao segundo plano. Com este artigo, Goodbody oferece importantes ferramentas àqueles que se interessam pelo assunto, disponibilizando uma larga e abrangente bibliografia sobre um tema relevante, mas ainda pouco abordado pela crítica literária alemã.

1 University of Bath (UK), Department of Politics, Languages \& International Studies. Email: a.h.goodbody@bath.ac.uk.

N. d. E.: Agradecemos a OUP pela permissão para publicar a tradução do artigo: GoODBODY, A., 2014. German ecocriticism : an overview. In: GARRARD, G., ed. The Oxford Handbook of Ecocriticism. Oxford, U. K.: Oxford University Press, pp. 547-559. Este texto possui a licença FREE TO VIEW ONLY; outros usos do mesmo devem obter a permissão da Oxford University Press e não são de responsabilidade da Revista Pandaemonium germanicum.

Pandaemonium, São Paulo, v. 17, n. 24, Dez. /2014, p. 1-19. 


\section{Goodbody, A. Ecocrítica alemã}

Ecocrítica é o termo utilizado para definir o estudo da relação entre a literatura e o meio-ambiente, posicionando a natureza em um ponto central dos interesses do homem. Esse debate é de grande importância na atualidade devido à necessidade de ações preventivas contra as mudanças climáticas. Embora os primeiros efeitos dessas mudanças já possam ser percebidos, um dos maiores impedimentos para a tomada de ações concretas continua sendo a incapacidade de as pessoas perceberem como essas mudanças podem afetar suas vidas cotidianas. A literatura exerce um importante efeito nesse sentido, transformando ameaças abstratas em possibilidades mais concretas e palpáveis para os seus leitores, como um alerta sobre atitudes que devem ser evitadas para que o pior não aconteça. Assim, é possível afirmar que, apesar de a ecocrítica ser especialmente destacada nos EUA, ela é uma área que deveria ser considerada relevante em todo o mundo.

Axel Goodbody nasceu em 1950 em Dublin. Estudou Germanística e Romanística no Trinity College em Dublin. Em 1983 defendeu seu doutorado acerca da lírica sobre a natureza no romantismo alemão e no século XX, e desde então leciona na Universidade de Bath. Foi um dos membros fundadores do braço britânico da ASLE (Association for the Study of Literature and Environment) em 1998 e da EASLCE (European Association for the Study of Literature, Culture and Environment) em 2004. Goodbody é considerado uma das principais autoridades na pesquisa de literatura ambiental alemã. É o autor de The Culture of German Environmentalism. Anxieties, Visions, Realities publicado em 2002, organizou, juntamente a Kate Ribgby, o livro Ecocritical Theory: New European Approaches em 2011, além de ter publicado diversos outros títulos e artigos sobre a questão ambiental tanto em língua inglesa quanto em alemão.

Esperamos, com esta tradução, oferecer subsídios e inspiração para os estudos ecocríticos no Brasil.

Valéria Sabrina Pereira

Universidade Federal de Minas Gerais

Pandaemonium, São Paulo, v. 17, n. 24, Dez. /2014, p. 1-19. 


\section{Goodbody, A. Ecocrítica alemã}

$\mathrm{O}$ contraste entre a resposta entusiástica da academia anglófona à ecocrítica e sua relativa invisibilidade no mundo falante de alemão é um verdadeiro enigma. ${ }^{2}$ Por que ela deveria ganhar mais reconhecimento como um campo de estudos literários na Alemanha, Áustria e Suíça, países em cuja filosofia e tradição cultural a natureza ocupa um lugar tão proeminente, cujo povo, segundo levantamentos internacionais de opinião pública, tem um alto índice de consciência ambiental, e onde as questões ambientais ocupam uma posição consistentemente alta na agenda política? Uma razão pode ser o fato de que cientistas, pensadores políticos e filósofos alemães têm sido pioneiros em ecologia desde Humboldt e Haeckel, e que livros não-ficcionais têm servido como o principal meio para o debate público sobre as questões ambientais na Alemanha. Há uma riqueza de pensamento ecológico cujas raízes se encontram na fenomenologia (de Heidegger aos Böhmes), no humanismo clássico (de Fromm a Jonas e Meyer-Abich) e na teoria social (da Escola de Frankfurt a Beck). Entretanto, a escrita literária alemã teve um impacto mais limitado no discurso ambiental e nas atitudes públicas, tanto em território nacional quanto no exterior.

Apesar de a maioria dos principais autores dos últimos quarenta anos (incluindo Christa Wolf, Hans Magnus Enzensberger e três ganhadores do prêmio Nobel, Günter Grass, Herta Müller e Elfriede Jelinek) terem tratado questões ambientais em algum ponto de suas obras, apenas um número relativamente pequeno de romances (ou filmes) importantes colocou as questões ambientais em primeiro plano na Alemanha desde um breve período que vai do início aos meados dos anos 1980. O escritor alemão cujo pensamento sobre o meio-ambiente goza de reconhecimento internacional é, de fato, Johann Wolfgang von Goethe, que escreveu durante o romantismo. ${ }^{3}$ Mesmo o projeto "Cultura e Clima" lançado pelo Instituto Goethe em 2009, como um foco temático especial de seu trabalho na representação dos interesses culturais da Alemanha no exterior, concentrou-se mais especialmente em artistas, fotógrafos, cineastas e artistas performáticos do que em escritores. Natureza e meio-ambiente certamente têm sido interesses destacados na arte alemã do século XX (dos artistas da colônia Worpswede e o expressionismo no início dos anos 1900 até Joseph Beuys e Friedensreich

\footnotetext{
${ }^{2}$ Gostaria de agradecer Gabriele Dürbeck, Agnes Kneitz, Bernhard Malkmus, Ute Seiderer, Berbeli Wanning e Evi Zemanek, que comentaram o esboço deste ensaio. Sem sua ajuda, eu estaria consideravelmente menos bem informado.

${ }^{3}$ Vide SEAMON; ZAJONC (1998), que compila ensaios sobre a perspectiva "ecológica" na ciência de Goethe e o seu uso contemporâneo (em especial, o ensaio de Nigel HofFMANN, "The Unity of Science and Art: Goethean Phenomenology as a New Ecological Discipline", 129-176). O trabalho de Peter SMITH também é importante nesse contexto (cf. SMITH 2000).
}

Pandaemonium, São Paulo, v. 17, n. 24, Dez. /2014, p. 1-19. 


\section{Goodbody, A. Ecocrítica alemã}

Hundertwasser), e discutivelmente também no cinema (se forem considerados os filmes alemães sobre montanhas, o Heimatfilm ${ }^{4}$, a obra de Werner Herzog, e muitos dos documentários sobre a natureza e filmes sobre a paisagem regional que têm sido exibidos na televisão no últimos anos). ${ }^{5}$ Contudo, permanece notório o fato de que artistas e cineastas hoje são considerados consideravelmente mais capazes do que os escritores alemães em auxiliar o público na reflexão sobre as causas humanas das mudanças climáticas e a imaginar suas consequências.

Uma outra razão para a relutância dos estudiosos de literatura na Alemanha a se engajarem na crítica focada na questão ambiental tem sido o legado de suspeita em relação ao sentimento "irracional” diante da natureza após 1945. Nos anos 80 e após, foi comum que esses estudiosos procurassem se distanciar do pensamento völkisch (i.e. racista-nacionalista) que emergiu por volta do final do século XIX e culminou no culto nazista de Blut und Boden (sangue e terra), e desconfiassem das conexões entre natureza e identidade nacional que frequentemente são encontradas em outras regiões. A carga ideológica, racialmente modulada, do pensamento alemão sobre a natureza, que havia sido alimentada por historiadores e críticos literários proeminentes nos anos 1930, pareceu para alguns refletir-se nos interesses temáticos centrais da ecocrítica, tais como a conservação da natureza e o pertencimento a um determinado lugar. Quando o movimento ambientalista surgiu na Alemanha, no início dos anos 1970, cerca de uma década depois do que nos EUA, sua mistura de argumentos racionais (muitas vezes simplistas) com uma oposição emocional aos valores materialistas e àquilo que foi percebido como tecnologias de alto risco, junto a sua retórica apocalíptica, pareceu para os acadêmicos um perigoso regresso ao romantismo e às formas antimodernistas da virada do século.

A história ambiental, entretanto, floresceu na Alemanha a partir dos anos 1980. Tanto na Europa quanto na América, estudiosos sujeitaram a uma análise crítica extensiva as atitudes inconstantes em relação à natureza e os conceitos de gerenciamento apropriado do meio-ambiente nos países falantes de alemão (Cf. LEKAN 2004; MAUCH 2004; BlacKBOURN 2006) Estudos mais especializados tendiam a ser focados ou na terra natal (Heimat) e nos movimentos juvenis de retorno à natureza da

\footnotetext{
${ }_{5}^{4}$ Filmes nostálgicos relacionados à Terra Natal (N. d. T.)

${ }^{5}$ Estudos sobre a arte ambiental e de paisagens na Alemanha incluem Frank FINLAY (1997) e Jael LEHMANN (2012). Estudos relevantes a respeito do cinema alemão incluem trabalhos que tratam de filmes sobre paisagens montanhosas (RENTSCHLER 1990), o Heimatfilm rural (PALFREYMAN 2002, VON MOLTKE 2005),e os filmes de Werner Herzog (GANDY 1996).
}

Pandaemonium, São Paulo, v. 17, n. 24, Dez. /2014, p. 1-19. 
Goodbody, A. Ecocrítica alemã

virada do século XX (cf. Rollins 1997), e no III Reich (Império Alemão) (Cf. Biehl/StaudenMaier 1995, BrÜGgemeIER/ CiOC/ Zeller 2005), ou no movimento ambientalista (cf. DOMINICK 1992; RADKAU 2011). A história ambiental surgiu como um campo que abarca elementos da história das ideias e da história cultural, juntamente à história política e social, e à geografia cultural. Em alguns casos, a história literária tem sido incluída nos relatos históricos da cultura e da sociedade alemã: representações literárias e artísticas vêm sendo abordadas em uma série de monografias e coleções de ensaios (Cf. Sieferle 1984, GroH; Groh 1991 e 1996, KirChHofF; TrePl 2009). Ao mesmo tempo, um trabalho importante tem sido conduzido por filósofos alemães que estão revisando as percepções inconstantes sobre a natureza (cf. SIEFERLE 1984, GROH; GroH 1991 e 1996, KiRCHHOFF; TREPL 2009), e exploram a ética (Cf. KREBS 1999) e a estética ambiental (cf. BÖHME 1989, VIETTA 1995, SEEL 1997). Linguistas, especialistas em estudos de mídia, psicólogos, etnólogos e teóricos políticos, todos eles continuaram produzindo estudos de relevância para a ecocrítica. Infelizmente, não seria possível fazer justiça à contribuição dessas disciplinas para a causa da ecocrítica dentro do escopo deste artigo. ${ }^{6}$

O contraste entre a riqueza do estudo ecocrítico em um sentido mais amplo e o número relativamente baixo de estudiosos de literatura alemã que escolheram, como já foi indicado, dedicar-se especificamente a temas ambientais é chocante. (O número de estudiosos que rotulou o próprio trabalho como "ecocrítico" é ainda menor.). Uma grande porção deles, além de tudo, são Auslandsgermanisten, ou seja, estudiosos que trabalham no exterior (p. ex. Jost HERMAND, Bernhard MALKMUS, Heather SulLIVAN e Sabine Wilke nos EUA, Kate RigBy na Austrália, Axel GoodBody e Colin RioRdAN no Reino Unido, Serenella IOviNO na Itália, e Nevzat KAYA na Turquia). Alemães que fizeram contribuições significantes aos estudos ecocríticos, como Hannes Bergthaller, Catrin Gersdorf, Christa Grewe-VolpP, Sylvia MaYer e Hubert ZAPF, geralmente trabalham com literatura norte-americana ou britânica, e a primeira conferência ecocrítica na Alemanha, na qual a afiliação europeia da ASLE (European

\footnotetext{
${ }^{6} \mathrm{O}$ contexto institucional é um fator que levou à emergência da ecocrítica literária na Alemanha como um ramo dos estudos culturais - em vez de um campo autônomo de pesquisa literária -, e a seus fortes laços com outras disciplinas de humanas. O Rachel Carson Center (RCC) em Munique é uma localidade central para o estudo ecocrítico interdisciplinar na atualidade. Fundado em 2009 como uma iniciativa conjunta da Universidade de Munique e o Deutsches Museum (Museu Nacional Alemão de Tecnologia), o RCC se ocupa de todos os aspectos da interação entre agentes humanos e a natureza. Buscando fortalecer o papel das humanidades nos debates políticos e científicos atuais sobre o meio-ambiente, ele é liderado por historiadores, mas inclui entre seus afiliados estudiosos de literatura e cinema como Sylvia MAYER, Agnes KNeITZ e Alexa WeIK.
}

Pandaemonium, São Paulo, v. 17, n. 24, Dez. /2014, p. 1-19. 
Association for the Study of Literature, Culture and Environment) foi criada, foi hospedada no Departamento de Inglês da Universidade de Münster em 2004.

Estudos em ingleses - mais precisamente norte-americanos - abriram o caminho para a introdução da ecocrítica literária na Alemanha. (A disciplina desempenhou anteriormente um papel semelhante ao do pós-colonialismo.) Entretanto, isso deveria ser entendido como uma reflexão sobre a sedimentação da experiência histórica nacional na diversidade cultural, mais do que indicar que os departamentos de literatura mainstream na Alemanha não têm nada a contribuir com o conhecimento literário de orientação ambientalista. A diversidade cultural está presente em diferentes níveis. Em primeiro lugar, há diferenças entre os repertórios linguísticos e a ressonância que determinados termos possuem. Em segundo, há a discrepância entre a importância relativa da escrita literária sobre determinados temas e em determinados gêneros: por exemplo, as representações da natureza selvagem são menos comuns do que as de "paisagem cultural", e a escrita da natureza tem um papel muito menos significante na tradição cultural alemã do que na norte-americana. De fato, ela sequer é reconhecida como um gênero. Por fim, mas não menos importante, há assimetrias no discurso acadêmico e em sua base filosófica, na constelação de escolas de pensamento e nas abordagens teóricas rivais, e na emergência de conceitos, categorizações, questões e abordagens de pesquisa. Todos esses fatores levaram à busca de diferentes tendências na teoria cultural.

Entretanto, o fato de o influente Metzler Lexikon Literatur- und Kulturtheorie [Léxico Metzler de teoria literária e da cultura], de Ansgar NÜNNING, contar com um verbete sobre "ecocrítica" já a partir de sua segunda edição (cf. HEISE 2001) pode ser visto como uma indicação de que a abordagem não é mais completamente desconhecida pelos estudantes alemães de teoria literária. De fato, a ecocrítica alemã começou, lentamente, a ganhar reconhecimento internacional. As contribuições alemãs para a filosofia da natureza, o pensamento ecológico e o estudo do investimento de uma significação simbólica da natureza na cultura popular foram largamente reconhecidas no exterior (cf. WORSTER 1977; HARRISON 1992; SCHAMA 1995), e a recente introdução à literatura e ao meio-ambiente de Timothy CLARK (2011) discute uma obra de escrita ambientalista alemã e cita a ecocrítica alemã (pela primeira vez em um livro inglês do gênero). Além de apresentar a novela de Wilhelm RAABE, Pfisters Mühle (1884, O Moinho de Pfister) e as obras pioneiras de ecojustiça (CLARK 2011: 96-8), Clark 


\section{Goodbody, A. Ecocrítica alemã}

examina a crítica de HEIDEGGER à tecnologia moderna (ibidem: 55-60), a estética de Gernot BöHME (ibidem: 81-2) e a teoria da literatura como ecologia cultural desenvolvida por Hubert ZAPF (ibidem: 153-5).

Sem se identificar como ecocríticos, os críticos literários alemães exploraram o rico campo alemão de representações literárias, artísticas e culturais de nosso relacionamento com o meio-ambiente, questionaram que contribuição deram romancistas, ensaístas, dramaturgos e poetas, diretores de cinema e artistas para reavaliá-la e para imaginar alternativas, e analisaram seus meios de produção e adaptação da tradição cultural. Essa obra inclui artigos e livros que já haviam sido publicados nos anos 1960 e 1970 sobre os idílios barrocos (cf. GARBER 1974), a psicoteologia e seu reflexo na poesia sobre a natureza do século XVIII (cf. KETELSEN 1974), o conceito de natureza de Goethe (cf. ZimMERMANN, 1969), a imagem da natureza no romantismo (cf. VON BORMANN 1968) e a poesia sobre a natureza moderna (cf. SCHÄFER 1969). Então surgiram as primeiras antologias sobre a literatura ambientalista no final dos anos 1970 e, com elas, artigos pioneiros que abordavam textos do cânone literário a partir da preocupação com o ambiente: Leo KREUTZER (1978) clamou por uma nova leitura dos poemas sobre a natureza de Goethe, e Horst DENKLER (1980) apontou o acima mencionado Pfisters Mühle de Raabe como um dos primeiros exemplos de reflexão sobre as consequências sociais e culturais da poluição industrial. Do início dos anos 1980 em diante, surgiu uma série de estudos, tais como as considerações de Wolfgang HERLE (1982) sobre a relação entre a humanidade e a natureza nos romances desde 1945, o estudo de Jürgen HAUPT (1982) abordando os poemas alemães sobre a natureza escritos no século XX, e o artigo de Hubertus KNABE (1985) e o livro de Anita MALLINCKRODT (1987), ambos sobre as representações do impacto da industrialização nos romances da RDA. ${ }^{7}$

Uma lista de títulos genuinamente ecocríticos, no sentido estrito de serem substancialmente focados seja na literatura alemã ou na teoria literária e guiados pelas preocupações com o meio-ambiente, se limitaria a uma dúzia de monografias e um número equivalente de volumes editados. Pode-se considerar a coleção de ensaios sobre representações literárias acerca da natureza e a naturalidade realizada por Reinhold GRIMM e Jost HERMAND (1981) como o primeiro deles. Hermand deu sequência a esse

\footnotetext{
${ }^{7}$ Afirmações programáticas sobre a necessidade examinar as representações literárias do meio-ambiente continuaram a surgir nos anos 1980 e 1990 (cf. BÖHME 1988 e 1994; HERMAND 1997).

Pandaemonium, São Paulo, v. 17, n. 24, Dez. /2014, p. 1-19.
} 
Goodbody, A. Ecocrítica alemã

estudo com uma monografia inovadora, Grüne Utopien in Deutschland (Utopias Verdes na Alemanha, 1991), uma brochura escrita para o público em geral que revisitou o pensamento verde na Alemanha desde Rousseau a partir de um ponto de vista ecossocialista. Através da discussão sobre a ficção, a poesia canônica, e muitos autores esquecidos, juntamente a ensaios e manifestos políticos, Hermand revelou a riqueza da tradição intelectual à qual escritores contemporâneos associados ao movimento ambiental poderiam se associar.

O primeiro estudioso que trabalha na Alemanha a publicar um estudo ocupando um livro completo, dessa vez não da perspectiva da tradição literária, mas da perspectiva da história das ideias, foi Gerhard KAISER. Seu livro Mutter Natur und die Dampfmaschine (Mãe natureza e a máquina a vapor, 1991) examinou a idealização da natureza que acompanhou a crescente objetivação científica e a dominação tecnológica do mundo natural como um fenômeno complementar do início do século XIX, e argumentou que os textos literários (de Goethe, Keller e Raabe) tiveram um papel fundamental em promover a influente figura da "mãe natureza". A primeira publicação significante em inglês foi a coleção de ensaios Green Thought in German Culture, editada por Colin Riordan em 1997, que surgiu como resultado de uma conferência de germanistas britânicos em Swansea. O volume combinou a revisão histórica com contribuições sobre o movimento ambientalista no início do século $\mathrm{XX}$, as dimensões ecológicas da teoria crítica, a religiosidade New Age e a política de direita, e ensaios sobre a escrita da Alemanha Ocidental e Oriental, a literatura suíça, as artes e o cinema. A produção literária de Carl Amery, romancista bávaro, comentarista cultural, pensador e ativista verde, foi o principal foco de um volume de ensaios multidisciplinar intitulado The Culture of German Environmentalism (GOODBODY 2002). Nele foram justapostos relatos sobre a história, a composição sociológica e as fundações teóricas do movimento ambientalista desde os anos 1970 com contribuições sobre o jornalismo, literatura e cinema alemães.

Enquanto isso, a germanista e comparativista australiana Kate RIGBY apresentou um relato comparativo magistral sobre a percepção do lugar da humanidade no mundo natural nos romantismos alemão e inglês em Topographies of the Sacred (2004). Rigby mostrou como Goethe, Novalis, Tieck e Eichendorff registraram e refletiram sobre o duplo empobrecimento da humanidade que foi resultado da demanda de limitarmos a nossa imaginação e a capacidade de ter empatia com as outras espécies da natureza, e da

Pandaemonium, São Paulo, v. 17, n. 24, Dez. /2014, p. 1-19. 
Goodbody, A. Ecocrítica alemã

rejeição do próprio aspecto corporal em prol da natureza mecânica. Sua obra está localizada dentro do contexto do romantismo continental e seus contemporâneos ingleses. Com base em Song of the Earth de Jonathan Bate, e informada por leituras pós-heideggerianas, Rigby confirma a importância do conceito de moradia para a análise ecocrítica, enquanto introduz modificações significantes. ${ }^{8}$

Em 2005 e 2006, foram publicados dois significantes volumes com artigos da primeira conferência sobre ecocrítica na Alemanha. Natur - Kultur - Text (Natureza Cultura - Texto, GERSDORF/ MAYER 2005) e Nature in Literary and Cultural Studies (GERSDORF/ MAYER 2006) combinaram explorações da teoria ecocrítica com análises textuais. O primeiro contém uma introdução à teoria ecocrítica para leitores falantes de alemão tratando a teoria cultural, a comunicação ambientalista e autores alemães desde Kleist. O volume em inglês é aberto por uma introdução à ecocrítica com ênfase na teoria da ecologia cultural. Os ensaios subsequentes abordam principalmente a literatura norte-americana, mas as contribuições de Riordan, Meacher, Griffiths e Goodbody tratam de textos alemães. ${ }^{9}$ A exposição de Stefan HoFER de uma teoria de sistemas ecocríticos, um estudo extenso publicado em 2007, levou Niklas LUHMANN a providenciar um fundamento teórico à função social da literatura, o qual se fazia ausente na pesquisa ecocrítica prévia. A insistência de Luhmann na separação na sociedade dos sistemas político, econômico, legal e cultural, e na relativa impossibilidade de eles influenciarem um ao outro, é concebida como uma forma de evitar tanto os argumentos normativos quanto a armadilha de confiar na exortação moral para resolver problemas. BERGTHALLER (2011) recentemente apresentou uma versão em língua inglesa dessa abordagem da teoria de sistemas. O livro de Axel Goodbody Nature, Technology and Cultural Change in $20^{\text {th }}$-Century German Literature (2007) é aberto por uma introdução à questão da natureza e do ambiente na cultura alemã, e às abordagens ecocríticas norteamericana, britânica e alemã. Na sequência, há um capítulo sobre o legado de Goethe. O livro traça então as mudanças ocorridas na atitude com relação ao meio-ambiente no decorrer do século XX através de estudos comparativos de obras sobre quatro temas: desastres tecnológicos, habitação, caça e a cidade. A coleção mais recente, Ökologische

\footnotetext{
${ }^{8}$ Heather Sullivan é responsável por outros estudos inovadores sobre Goethe e o romantismo (cf. SULLIVAN 2003 e 2010).

9 As teses de doutorado não publicadas de Hope, Meacher e Griffiths, e o estudo da escrita contemporânea suíça de Andrew LISTON (2011) são outros exemplos da ecocrítica germanista britânica. A tradição literária suíça, na qual a paisagem dos alpes tem servido como foco para as reflexões sobre o sublime, a vida simples e o impacto danoso da modernização, também tem sido objeto de estudos, incluindo Jürgen BARKHOFF (1997), Sean IRETON e Caroline SCHAUMANN (2012).
}

Pandaemonium, São Paulo, v. 17, n. 24, Dez. /2014, p. 1-19. 
Transformationen und literarische Repräsentationen (Transformações ecológicas e representações literárias, ERMISCH 2010) contém ensaios originados em um simpósio realizado por especialistas em literatura alemã (com o suporte de historiadores ambientais) na Universidade de Göttingen. Ela pode ser vista como o marco da derradeira aceitação da ecocrítica nos estudos literários alemães mainstream. ${ }^{10} \mathrm{O}$ volume reúne contribuições cuidadosamente fundamentadas sobre autores clássicos, modernos e contemporâneos, e sobre gêneros que vão de poesia e escrita sobre a natureza à literatura infantil e ecoficção. ${ }^{11}$

Na Alemanha, assim como em todo lugar, a pastoral e o apocalipse serviram como modalidades de produção cultural centrais para as representações do meioambiente. Nos anos 1970, a Heimat (ou terra natal) era redefinida e reabilitada pelas pessoas originárias de um local no contexto do movimento ambientalista. Romances e filmes refletiram esse processo, um exemplo é Heimat de Edgar Reitz (que se desenvolveu, desde suas primeiras séries em 1984, de forma a se tornar um épico de 53 horas que traça a vida em um vilarejo rural de 1919 a 2000). Os estudos críticos de Heimat e suas representações literárias e visuais (cf. BLICKLE 1992; BOA/ PALFREYMAN 2000) incluíram cada vez mais reflexões sobre o papel daqueles que são pertencentes a um local e a motivação para conduzir um estilo de vida sustentável (cf. GoODBODY (2013). A topografia literária surgiu como um foco para as contribuições alemãs dedicadas à pesquisa orientada pela psicologia. As representações de paisagens como um repositório de experiência histórica foram objeto de análise crítica (sendo que a ênfase normalmente se dá na violência política e na destruição do meio-ambiente) na obra de Wolf e Sebald, e dos austríacos Bachmann, Bernhard, Handke e Jelinek, por exemplo. $^{12}$

A partir dos anos 1980, os estudos de literatura apocalíptica focalizaram, de modo crescente, a representação de catástrofes ambientais (cf. p. ex. GROH/KeMPER/MAUleshagen 2003, e RigBy 2008). A mudança climática levou a um grande aumento no interesse pelo tópico nos últimos anos (cf. DüRBECK 2012, e MAUCH/ MAYER 2012). A mudança climática na literatura alemã também é uma área de

${ }^{10} \mathrm{O}$ principal organizador do simpósio foi Heinrich Detering, cujo interesse de longa-data nas sutilezas da reflexão literária sobre questões ambientais é evidenciado por DETERING 1992 e 2008.

${ }^{11}$ Gêneros de prosa popular que atraíram atenção da crítica incluem o eco-thriller (cf. WANNING 2008), romances policiais (cf. SCHÜLLER 1997), ficção científica (cf. STAPLETON 1993) e narrativas de risco (cf. HEISE 2008; ZEMANEK 2012).

${ }_{12}$ Estudos-chave da topografia literária alemã incluem WeIGEL (1996), Hartmut BöHME (2005) e WEBBER (2008). 
interesse do "Climate Culture", grupo conduzido pelo Institute for Advanced Studies in the Humanities em Essen, e uma das linhas de pesquisa do Environmental Humanities Transatlantic Research Network fundado pela Humboldt Foundation e guiado por Sabine Wilke em Seattle.

Representações e reflexões sobre desastres naturais, estágios da destruição humana e processos naturais de decadência têm sido um traço da escrita alemã desde a Segunda Guerra, de Arno Schmidt a W.G. Sebald; a obra ricamente complexa de Sebald serviu como um vínculo entre o interesse pela ecocrítica, os estudos de memória cultural, a escrita de viagens, a autobiografia e a literatura do holocausto (especialmente em seu longo poema Do Natural e no relato sobre um passeio a pé por Sussex, em Os anéis de Saturno). ${ }^{13}$ Questões de justiça e racismo ambiental têm sido tratadas de forma oblíqua na Alemanha através de representações da desterritorialização e expropriação dos judeus, resultando em uma dimensão ecocrítica em algumas obras do campo de estudos do holocausto. Em Sebald, há mais um elo entre os dois objetos: a posição distinta do narrador à margem, procurando por uma identificação tática com seus protagonistas judeus, é refletida na forma como Sebald dá voz a animais e à natureza como vítimas da devassa destruição humana. Em uma das contribuições de orientação crítica mais intrigantes das pesquisas sobre Sebald, que crescem rapidamente desde a morte prematura do autor em 2001, On Creaturely Life (2006), Eric SANTNER toma a redefinição de Agamben do conceito teológico de "criatura" como uma categoria biopolítica, na qual o ser humano é reduzido ao estado de passividade, de ser perpetuamente criado, sob as traumáticas condições do governo soberano arbitrário e a violência institucional na modernidade. Santner lê a prosa de Sebald como um local para a exploração do reino de sofrimentos da criatura como resultado do holocausto.

Um panorama desse tipo não estaria completo sem que se procurasse oferecer uma visão mais geral dos desenvolvimentos na teoria ecocrítica na Alemanha, além de identificar a contribuição alemã. Durante a propagação da abordagem ecocrítica do mundo anglófono para outros países e comunidades acadêmicas, ocorrida na última década, estudiosos alemães, assim como os de outras regiões, ligaram-se a tradições predominantemente locais, diversificando e enriquecendo a abordagem ecológica nesse processo. Os debates teóricos alemães nos anos setenta e oitenta foram dominados menos pelo pós-modernismo e pelo pós-estruturalismo do que pela hermenêutica com

\footnotetext{
${ }^{13}$ Publicações sobre a natureza na escrita de Sebald incluem Fuchs (2007) e MALKMUS (2011).

Pandaemonium, São Paulo, v. 17, n. 24, Dez. /2014, p. 1-19.
} 


\section{Goodbody, A. Ecocrítica alemã}

base em Gadamer, nas abordagens neomarxistas da Escola de Frankfurt por Adorno e Benjamin, e na antropologia cultural (especialmente na teoria da recepção de Wolfgang Iser e na obra de Jan e Aleida Assmann sobre a memória cultural). É natural que a ecocrítica alemã tenha sido influenciada por essas correntes de pensamento.

Tino MüLLER recentemente (2011) argumentou que dois dos principais modelos da teoria ecocrítica alemã atuais têm suas raízes na antropologia literária. ${ }^{14}$ Nos anos 1980, Wolfgang Iser desenvolveu um conceito da função dos textos literários que se baseia em seu potencial de contrastar experiências diárias com possíveis alternativas ficcionais, permitindo que os leitores desenvolvam e modifiquem seu autoentendimento ao cruzar os limites em um processo imaginativo. Gernot e Hartmut BöHME pensaram subsequentemente nas consequências ecológicas dessa abordagem. Sua "estética da natureza" é fundada no humanismo liberal tradicional, mas o molda através da ideia de uma sensibilidade especial que permita que os seres humanos voltem a se conectar com a natureza.

Para estabelecer relações novas e não hierárquicas com a natureza, os autores argumentam que precisamos revisitar conceitos simbióticos pré-modernos do ser humano em seu ambiente natural, tais como a ideia de Paracelso de uma "linguagem da natureza" simbiótica. Ela pode ter sido suplantada nas ciências naturais, mas continuou sendo uma força produtiva na história das ideias, contribuindo tanto com a literatura (Novalis, Baudelaire e grande parte da poesia sobre a natureza do século XX) quanto com a filosofia (Kant, Benjamin, Adorno, Blumenberg). Além disso, Gernot e Hartmut Böhme clamam que é através de nossas sensações físicas e reações ao meio-ambiente que nos comunicamos com o mundo objetivo. ${ }^{15}$ Traços da experiência física estão presentes em toda a linguagem, mas são mais palpáveis nos textos poéticos que trabalham com metáforas e imagens e consequentemente têm um papel especial como um meio que facilita se reconectar com a natureza. Em uma era de destruição ambiental, o arquivo cultural de textos literários é um recurso cujo potencial não deveria ser negligenciado em estratégias de renaturalização. A literatura grava e guarda informações sobre como as sociedades se posicionam em relação à natureza, dando voz

\footnotetext{
${ }^{14}$ Não se deve negar que outros aspectos da teoria literária amplamente utilizados por estudiosos alemães (p. ex. marxismo e psicanálise) possuem uma dimensão ecocrítica. Entretanto, tem havido uma ausência notável de contribuições ecofeministas, e, enquanto Heidegger continua sendo um ponto de referência central para a ecocrítica internacional, essa crítica da tecnologia raramente é citada como modelo pelos críticos literários alemães.

15 A teoria das "atmosferas" de Gernot Böhme (cf. RigBY 2011) é a principal forma na qual a fenomenologia está presente na teoria ecocrítica alemã.
}

Pandaemonium, São Paulo, v. 17, n. 24, Dez. /2014, p. 1-19. 
a aspectos da cultura que, de outra maneira, são excluídos e silenciados, tais como as mulheres, os povos "não-civilizados" e o mundo físico. A sobrevivência da raça humana depende da reinstituição de sensibilidades ameaçadas como prioridade máxima. Hartmut Böhme traça um elo entre esse papel de facilitar a sobrevivência humana desempenhado pela literatura, a arte e a estética, e o conceito de natureza como um “projeto cultural” (BÖHME/MATUSSEK/MÜLLER 2000: 118-31). Devemos aceitar a responsabilidade por moldá-la, conscientes de que o nosso controle sobre ela não é ilimitado. Obras de arte podem tanto servir como modelo estético de interação humana com a natureza, quanto imaginar e representar utopias alternativas aos padrões contemporâneos de comportamento.

A segunda contribuição significante à teoria ecocrítica, a fusão da ecologia cultural e do criticismo textual desenvolvida por Hubert ZAPF (2002), considera os textos literários capazes de revitalizar o sistema cultural através de condensar e transformar elementos do discurso público em construtos nodais, como símbolos e metáforas. Enquanto Böhme permanece centrado no objeto em sua tentativa de superar os problemas associados ao antropocentrismo, Zapf adota uma abordagem sistêmica, questionando que função a cultura desempenha na sociedade. Ele faz uma distinção entre três funções discursivas da literatura igualmente importantes como um meio da ecologia cultural: uma função crítica à cultura, uma imaginativa e uma reintegrativa (ZAPF 2002: 33-9). Primeiro, a literatura chama a atenção para estruturas opressivas do sistema cultural. Em seguida, ela dá voz àquilo que é suprimido por essas culturas e providencia uma base de testes para formas alternativas de organização cultural. E, por fim, ela tem uma capacidade única de tratar da pessoa como um todo, cruzar fronteiras entre sistemas sociais e discursos que, em outras situações, se encontram divididos. $\mathrm{O}$ impacto cultural dos textos literários provém, acima de tudo, de sua condensação de informações tanto simbólica quanto metafórica.

Como deveriam ser resumidas as realizações e contribuições à ecocrítica alemã feitas até hoje? Talvez afirmando que ela se baseou em e explicou pensamentos que compartilham muito com a cultura norte-americana e britânica, mas possivelmente se diferencia na maneira como instrui sobre os problemas ambientais. Através de comparações interdisciplinares e interculturais teoricamente informadas, a ecocrítica alemã também adicionou uma série de perspectivas e metodologias à caixa de ferramentas da comunidade internacional de pesquisadores. Meu ponto de partida foi o 
Goodbody, A. Ecocrítica alemã

fato de que a crítica literária parece ter ocupado um papel menos proeminente nos debates sobre a sustentabilidade na Alemanha do que nos EUA, o que pode ser um reflexo do domínio dos discursos filosófico, ético, histórico, político e social, assim como do volume relativamente modesto e do status da escrita literária alemã sobre o meio-ambiente. O pensamento ecológico deve ser centrado na literatura? Talvez haja razões históricas e culturais especiais que justifiquem que as coisas sejam assim nos EUA, e isso deveria ser considerado como a exceção mais do que como a regra.

\section{Referência Biográficas}

BARKHOFF, Jürgen. Green Thought in Modern Swiss Literature. In: RIORDAN, Colin (ed.). Green Thought in German Culture. Historical and Contemporary Perspectives. Cardiff: University of Wales Press, 1997, 223-41.

Bergthaller, Hannes. Cybernetics and Social Systems Theory. In: GoOdBody, Axel; Rigby, Kate (ed.). Ecocritical Theory: New European Contributions. Charlottesville and London: University of Virginia Press, 2011, 217-29.

BieHl, Janet; StaudenMAIER, Peter. Ecofascism. Lessons from the German Experience. Edinburgh and San Francisco: AK Press, 1995.

Blackbourn, David. The Conquest of Nature. Water, Landscape, and the Making of Modern Germany. London: Jonathan Cape, 2006.

BLICKLE, Peter, Heimat. A Critical Theory of the German Idea of Homeland. Rochester, New York: Camden House, 1992.

BoA, Elizabeth; Palfreyman, Rachel. Heimat. A German Dream. Regional Loyalties and National Identity in German Culture 1890-1990. Oxford: Oxford University Press ,2000.

BÖHME, Gernot. Für eine ökologische Naturästhetik. Frankfurt am Main: Suhrkamp, 1989.

. Natürlich Natur. Über Natur im Zeitalter ihrer technischen Reproduzierbarkeit. Frankfurt am Main: Suhrkamp, 1992.

BöHME, Hartmut (ed.). Kulturgeschichte des Wassers. Frankfurt am Main: Suhrkamp, 1988.

. Natur und Subjekt. Frankfurt am Main: Hartmut Böhme, 1988

Literaturwissenschaft in der Herausforderung der technischen und ökologischen Welt. In JäGER, Ludwig; SwITALLA, Bernd (ed.). Germanistik in der Mediengesellschaft. München: Fink, 1994, 63-79.

. (ed.). Topographien der Literatur: Deutsche Literatur im transnationalen Kontext. Stuttgart: Metzler, 2005.

BÖHME, Hartmut, MATUSSEK, Peter; MÜLlER, Lothar. Orientierung Kulturwissenschaft. Was sie kann, was sie will. Reinbek bei Hamburg: Rowohlt, 2000.

BRAUNGART, Georg and Urs Büttner (ed.), Wind und Wetter: Kultur - Wissen - Ästhetik. München: Wilhelm Fink Verlag, 2014.

BRÜGGEMEIER, Franz-Josef; CIOC, Mark; ZELLER, Thomas (ed.). How Green Were the Nazis? Nature, Environment, and Nation in the Third Reich. Athens, GA: Ohio University Press, 2005. 


\section{Goodbody, A. Ecocrítica alemã}

ClaRK, Timothy. The Cambridge Introduction to Literature and the Environment. Cambridge: Cambridge University Press, 2011.

DENKLER, Horst. Nachwort. In: RAABE, Wilhelm. Pfisters Mühle. Ein Sommerferienheft, Stuttgart: Reclam, 1980, 225-51.

DETERING, Heinrich. Ökologische Krise und ästhetische Innovation im Werk Wilhelm Raabes. Jahrbuch der Raabe-Gesellschaft, 1992, 1-27.

"So könnte die Welt untergehen": Ökologie und Literatur im 18. Jahrhundert, Lichtenberg-Jahrbuch, 2008, 7-20.

DominicK, Raymond H. The Environmental Movement in Germany. Prophets and Pioneers, 1871-1971. Bloomington; Indianapolis: Indiana University Press, 1992.

DÜRBECK, Gabriele (ed.) Writing Catastrophes: Cross-disciplinary Perspectives on the Semantics of Natural and Anthropogenic Disasters (Ecozon@ 3:1 [Spring 2012]).

"Popular Science and Apocalyptic Narrative in Frank Schätzing's The Swarm". In Dürbeck, 2012, 20-30.

ERMisCh, Maren, KRUSE, Ulrike; STOBBE, Urte (ed.). Ökologische Transformationen und literarische Repräsentationen. Veröffentlichungen des Graduiertenkollegs Interdisziplinäre Umweltgeschichte. Göttingen: Universitätsverlag Göttingen, 2010.

FINLAY, Frank. Joseph Beuys' Eco-Aesthetics. In: RIORDAN, Colin (ed.). Green Thought in German Culture. Historical and Contemporary Perspectives. Cardiff: University of Wales Press, 1997, 245-58.

FuCHS, Anne. "Ein Hauptkapitel der Geschichte der Unterwerfung": Representations of Nature in W.G. Sebald's Die Ringe des Saturn. In: FuCHS, Anne; LONG, J.J. (ed.). W.G. Sebald and the Writing of History. Würzburg: Königshausen and Neumann, 2007, 121-38.

GANDY, Matthew. Visions of Darkness: The Representation of Nature in the Films of Werner Herzog. In: Ecumene 3:1 (1996), 1-21.

GARBER, Klaus. Der locus amoenus und der locus terribilis. Bild und Funktion der Natur in der deutschen Schäfer- und Landlebendichtung des 17. Jahrhunderts. Köln, Wien: Böhlau, 1974.

Gersdorf, Catrin; MAYER, Sylvia (ed.). Natur - Kultur - Text. Beiträge zu Ökologie und Literaturwissenschaft. Heidelberg: Universitätsverlag Winter, 2005.

(ed.). Nature in Literary and Cultural Studies. Transatlantic Conversations on Ecocriticism. Amsterdam and New York: Rodopi, 2006.

Goodbody, Axel (ed.). The Culture of German Environmentalism. Anxieties, Visions, Realities. New York and Oxford: Berghahn ,2002.

. (ed.) Literatur und Ökologie. Amsterdam: Rodopi, 1998.

. (ed.) Nature, Technology and Cultural Change in Twentieth-Century German Literature. The Challenge of Ecocriticism. Basingstoke: Palgrave Macmillan, 2007.

. Heimat als Identität und ökologisches Bewusstsein stiftender Faktor: Zu Ansätzen in Romanen um 1900 von Bruno Wille, Hermann Hesse und Josef Ponten. In: PAULSEN, Adam; SANDBERG, Anna (ed.). Natur und Moderne um 1900. Räume Repräsentationen. Bielefeld: transcript, 2013, 183-202.

Melting ice and the paradoxes of Zeno: Didactic impulses and aesthetic distanciation in "German climate change fiction”.Ecozon@ 4 :1 (Spring 2013), 92-102.

Goodbody, Axel; Rigby, Kate (ed.). Ecocritical Theory: New European Contributions. Charlottesville, London: University of Virginia Press, 2011.

GoodBody, Axel and Berbeli Wanning (ed.), Wasser - Kultur - Ökologie: Konstanten und Wandel in der sozialen und kulturellen Bedeutung des flüssigen Elements, Göttingen: V\&R unipress, 2008. 
Goodbody, A. Ecocrítica alemã

GRIMM, Reinhold; HERMAND, Jost (ed.). Natur und Natürlichkeit. Stationen des Grünen in der deutschen Literatur. Königstein im Taunus: Athenäum, 1981.

GROH, Ruth; GROH, Dieter. Zur Kulturgeschichte der Natur (2 vols). Frankfurt am Main: Suhrkamp, 1991 e 1996.

GrOH, Dieter, KEMPE, Michael; MAUlEShagen, Franz (ed.). Naturkatastrophen: Beiträge zu ihrer Deutung, Wahrnehmung und Darstellung in Text und Bild von der Antike bis ins 20. Jahrhundert. Tübingen: Gunter Narr ,2003.

HARrison, Robert Pogue. Forests. The Shadow of Civilization. Chicago and London: University of Chicago Press, 1992.

HAUPT, Jürgen. Natur und Lyrik. Naturbeziehungen im 20. Jahrhundert. Stuttgart: Metzler, 1982.

HEISE, Ursula. Ecocriticism/ Ökokritik. In: NÜNNING, Ansgar (ed.). Metzler Lexikon Literaturund Kulturtheorie. Stuttgart ,Weimar: Metzler, $2^{\mathrm{a}}$ ed., 2001, 128-9.

. Sense of Place and Sense of Planet: The Environmental Imagination of the Global. Oxford: Oxford University Press, 2008.

_. Nach der Natur. Das Artensterben und die moderne Kultur, Frankfurt am Main: Suhrkamp, 2010.

HERLES, Wolfgang. Der Beziehungswandel zwischen Mensch und Natur im Spiegel der deutschen Literatur seit 1945. Stuttgart: Heinz, 1982.

HERMAND, Jost. Grüne Utopien in Deutschland. Zur Geschichte des ökologischen Bewusstseins. Frankfurt am Main: Fischer, 1991.

Im Wettlauf mit der Zeit : Anstösse zu einer ökologiebewussten Ästhetik. Berlin: edition sigma, 1991.

Literaturwissenschaft und ökologisches Bewusstsein. Eine mühsame Verflechtung. In BENTFEld, Anne; Delabar, Walter (ed.). Perspektiven der Germanistik. Neueste Ansichten zu einem alten Problem. Opladen: Westdeutscher Verlag, 1997, 106-25.

HOFER, Stefan. Die Ökologie der Literatur. Eine systemtheoretische Annäherung. Mit einer Studie zu Werken Peter Handkes. Bielefeld: transcript, 2007.

HoRN, Eva. Enden des Menschen. Globale Katastrophen als biopolitische Fantasie. In: SoRG, Reto; WÜRFFEL, Stefan Bodo (ed.). Utopie und Apokalypse in der Moderne. München: 2010, 101-18.

Ireton, Sean; Schaumann, Caroline (ed.). Heights of Reflection. Mountains in the German Imagination from the Middle Ages to the Twenty-First Century. Rochester, New York: Camden House, 2012.

Jael Lehmann, Annette. Environments: Künste Medien Umwelt. Facetten der künstlerischen Auseinandersetzung mit Landschaft und Natur. Bielefeld: transcript, 2012.

KAISER, Gerhard. Mutter Natur und die Dampfmaschine. Ein literarischer Mythos im Rückbezug auf Antike und Christentum. Freiburg im Breisgau: Rombach, 1991.

KeTELSEN, Uwe-Karsten. Die Naturpoesie der norddeutschen Frühaufklärung. Poesie als Sprache der Versöhnung, alter Universalismus und neues Weltbild. Stuttgart: Metzler, 1974.

KIRCHHOFF, Thomas; TREPL, Ludwig (ed.). Vieldeutige Natur: Landschaft, Wildnis und Ökosystem als kulturgeschichtliche Phänomene. Bielefeld: transcript, 2009.

KNABE, Hubertus, Zweifel an der Industriegesellschaft. Ökologische Kritik in der erzählenden DDR-Literatur. In: Redaktion Deutschland Archiv (ed.). Umweltprobleme und Umweltbewußtsein in der DDR. Köln: Verlag Wissenschaft und Politik. 1985, 201-50.

KREBS, Angelika. Ethics of Nature. Berlin, New York: de Gruyter, 1999.

KREUTZER, Leo. Wie herrlich leuchtet uns die Natur?. In: Akzente 25:4 (1978), 381-90.

Pandaemonium, São Paulo, v. 17, n. 24, Dez. /2014, p. 1-19. 
Goodbody, A. Ecocrítica alemã

LEKAN, Thomas M. Imagining the Nation in Nature: Landscape Preservation and German Identity, 1885-1945. Cambridge, Mass. and London: Harvard University Press, 2004.

LILIENTHAL, Volker. Irrlichter aus dem Dunkel der Zukunft. Zur neueren deutschen Katastrophenliteratur. In: KREUZER, Helmut (ed.), Pluralismus und Postmodernismus. Zur Literatur- und Kulturgeschichte in Deutschland 1980-1995. $4^{\text {a }}$ ed. Frankfurt am Main: Peter Lang, 1996, 257-96.

LINDENPÜTZ, Dagmar. Das Kinderbuch als Medium ökologischer Bildung. Untersuchungen zur Konzeption von Natur und Umwelt in der erzählenden Kinderliteratur seit 1970. Essen: Die Blaue Eule, 1999.

Liston, Andrew. The Ecological Voice in Recent German-Swiss Prose. Oxford, etc.: Peter Lang, 2011.

MALKMUS, Bernhard. Das Naturtheater des W.G. Sebald: Die ökologischen Aporien eines poeta doctus. In: LÜTZELER, Paul Michael; MCGLOTHLIN, Erin (ed.). Gegenwartsliteratur. A German Studies Yearbook 10. Tübingen: Stauffenburg, 2011, 210-33.

MallinCKRODT, Anita. The Environmental Dialogue in the GDR. Literature, Church, Party and Interest Groups in their Socio-Political Context. A Research Concept and Case Study. Lanham: University Press of America, 1987.

MAUCH, Christoph (ed.). Nature in German History. New York and Oxford: Berghahn, 2004.

MAYER, Sylvia; MAUCH, Christoph (ed.). Green Cultures: Environmental Knowledge, Climate, and Catastrophe. Heidelberg: Universitätsverlag Winter, 2012.

MAYER, Sylvia; VON MOSSNER, Alexa Weik (ed.), The Anticipation of Catastrophe. Environmental Risk in North American Literature and Culture. Heidelberg: Winter Verlag, 2014.

MeCKLENBURG, Norbert. Die grünen Inseln. Zur Kritik des literarischen Heimatkomplexes. München: iudicium, 1987.

MEYER-ABICH, Klaus Michael. Revolution for Nature. From the Environment to the Connatural World. Cambridge: White Horse Press, 1993

MÜLLER, Timo. From Literary Anthropology to Cultural Ecology: German Ecocritical Theory since Wolfgang Iser In: GoODBODY, Axel; Rigby, Kate (ed.). Ecocritical Theory: New European Contributions. Charlottesville and London: University of Virginia Press, 2011, 71-83.

MÜLleR, Timo; SAUTER, Michael (ed.). Literature, Ecology, Ethics: Recent Trends in Ecocriticism. Heidelberg: Universitätsverlag Winter GmbH Heidelberg, 2012.

PALFREYMAN, Rachel. Green Strands on the Silver Screen? Heimat and Environment in the German Cinema. In: GoodBody, Axel (ed.). The Culture of German Environmentalism. Anxieties, Visions, Realities. New York and Oxford: Berghahn ,2002, 171-86.

RADKAU, Joachim. Die Ära der Ökologie. München: Beck, 2011.

RAPP, Friedrich (ed.). Naturverständnis und Naturbeherrschung. Philosophiegeschichtliche Entwicklung und gegenwärtiger Kontext. München: Fink, 1981.

RENTSCHLER, Eric. "Mountains and Modernity: Relocating the Bergfilm". In: New German Critique 51 (Autumn 1990), 137-61.

RIGBY, Kate. Topographies of the Sacred. The Poetics of Place in European Romanticism. Charlottesville, London: University of Virginia Press, 2004.

Discoursing on Disaster: The Hermeneutics of Environmental Catastrophe. In: Tamkang Review 39:1 (2008), 19-40.

Gernot Böhme's Ecological Aesthetics of Atmosphere. In: GoODBODY, Axel; Rigby, Kate (ed.). Ecocritical Theory: New European Contributions. Charlottesville and London: University of Virginia Press, 2011, 139-52. 
Goodbody, A. Ecocrítica alemã

RIORDAN, Colin (ed.). Green Thought in German Culture. Historical and Contemporary Perspectives. Cardiff: University of Wales Press, 1997.

Rollins, William H. A Greener Vision of Home. Cultural Politics and Environmental Reform in the German Heimatschutz Movement, 1904-1918. Michigan: University of Michigan Press, 1997.

SANTNER, Eric. On Creaturely Life. Rilke, Benjamin, Sebald. Chicago: University of Chicago Press, 2006.

SCHÄFER, Hans Dieter. Wilhelm Lehmann. Studien zu seinem Leben und Werk. Bonn: Bouvier, 1969.

SCHÄFER, Lothar. Das Bacon-Projekt. Von der Erkenntnis, Nutzung und Schonung der Natur. Frankfurt am Main: Suhrkamp, 1993.

SCHAMA, Simon. Landscape and Memory. London: HarperCollins, 1995.

SCHARNOWSKI, Susanne. Literatur für das Anthropozän. Die Ästhetik der berührten Natur in Dieter Bachmanns Unter Tieren und Andreas Maiers/ Christine Büchners Bullau. Versuch über Natur. In: AMTHOR, Wiebke; HILlE, Almut; SCHARNOwSKI, Susanne (ed.). Wilde Lektüren. Literatur und Leidenschaft. Festschrift für Hans Richard Brittnacher zum 60. Geburtstag, Bielefeld: Aisthesis, 2012, 363-80.

SEAmon, David; Zajonc, Arthur (ed.). Goethe's Way of Science. A Phenomenology of Nature. New York: State University of New York Press, 1998.

SEEL, Martin. Eine Ästhetik der Natur. Frankfurt am Main: Suhrkamp ,1991.

SIEFERLE, Rolf Peter (ed.). Fortschrittsfeinde? Opposition gegen Technik und Industrie von der Romantik bis zur Gegenwart. München: Beck, 1984.

SMITH, Peter D. Metaphor and Materiality: German Literature and the Worldview of Science: 1780-1955. Oxford: Legenda, 2000.

Stapleton, Amy. Utopias for a Dying World. Contemporary German Science Fiction's Plea for a New Ecological Awareness. New York, etc.: Peter Lang, 1993.

SulLIVAN, Heather. Organic and Inorganic Bodies in the Age of Goethe: An Ecocritical Reading of Ludwig Tieck's Rune Mountain and the Earth Sciences. In: ISLE 10:2 (2003), 21-46.

Ecocriticism, the Elements, and the Ascent/ Descent into Weather in Goethe's Faust. In: Goethe Yearbook 2010, 55-72.

UTZ, Peter. Wenn in der Schweiz die Welt untergeht. Literatur aus der Schweiz und ihre Katastrophenszenarien. In: SORG, Reto; CADUFF, Corinna (ed.). Nationale Literaturen ein Phantom? Die Imagination und Tradition des Schweizerischen als Problem. München: Fink, 2004, 219-233.

VIETTA, Silvio. Die vollendete Speculation führt zur Natur zurück. Natur und Ästhetik. Leipzig: Reclam, 1995.

VON BORMANN, Alexander. Natura Loquitur. Naturpoesie und emblematische Formel bei Joseph von Eichendorff. Tübingen: Niemeyer, 1968.

von MoltKe, Johannes. No Place Like Home: Locations of Heimat in German Cinema. Berkeley, Los Angeles: University of California Press, 2005.

WANNING, Berbeli. Yrrsinn oder die Auflehnung der Natur - Kulturökologische Betrachtungen zu Der Schwarm von Frank Schätzing. In: Hubert Zapf et al. (ed.). Kulturökologie und Literatur. Beiträge zu einem neuen Paradigma der Literaturwissenschaft. Würzburg: Königshausen and Neumann, 2008, 339-57.

WeBber, Andrew. Berlin in the Twentieth Century. A Cultural Topography. Cambridge: Cambridge University Press, 2008.

WeIGEL, Sigrid. Body- and Image-Space. Re-reading Walter Benjamin. Trad.: Georgina Paul, Rachel McNicholl e Jeremy Gaines. London: Routledge, 1996. 
Goodbody, A. Ecocrítica alemã

WILKE, Joachim (ed.). Zum Naturbegriff der Gegenwart. Kongressdokumentation zum Projekt 'Natur im Kopf'. 2 vols. Stuttgart, Bad Cannstatt: Frommann-Holzboog, 1993.

WORSTER, Donald. Nature's Economy: A History of Ecological Ideas. San Francisco: Sierra Club, 1977.

ZAPF, Hubert. Literatur als kulturelle Ökologie: Zur kulturellen Funktion imaginativer Texte an Beispielen des amerikanischen Romans. Tübingen: Niemeyer, 2002.

ZEMANEK, Evi. Naturkatastrophen in neuen Formaten. Fakten und Fiktionen des Tsunami in Frank Schätzings Ökothriller Der Schwarm und Josef Haslingers Augenzeugenbericht Phi Phi Island. In: Bohley, Johanna; Schöll, Julia (ed.). Das erste Jahrzehnt. Narrative und Poetiken des 21. Jahrhunderts. Würzburg: Königshausen und Neumann, 2011, 8398.

Unkalkulierbare Risiken und ihre Nebenwirkungen. $\mathrm{Zu}$ literarischen Reaktionen auf ökologische Transformationen und den Chancen des Ecocriticism. In: SCHMITZEMANS, Monika et al. (ed.). Literatur als Wagnis/ Literature as Risk. DFG-Symposium. Berlin, Boston: de Gruyter: 2013, 279-302.

Zimmermann, Rolf Christian. Das Weltbild des jungen Goethe, München: Fink, 1969.

Recebido em 29/08/2014

aceito em 14/10/2014 